\title{
Health status, heat preparation strategies and medical events among elite cyclists who competed in the heat at the 2016 UCI Road World Cycling Championships in Qatar
}

\author{
Sebastien Racinais @ (1) , ${ }^{1,2}$ David Nichols, ${ }^{1}$ Gavin Travers, ${ }^{1}$ Sebastien Moussay, ${ }^{3}$ \\ Taoufik Belfekih, ${ }^{1}$ Abdulaziz Farooq (1) , ${ }^{1}$ Yorck Olaf Schumacher, ${ }^{1}$ Julien D Périard ${ }^{1,4}$
}

${ }^{1}$ Aspetar Orthopaedic and Sports Medicine Hospital, Doha, Qatar

'Laboratory Sport, Expertise and Performance, French Institute of Sport (INSEP), Paris, France ${ }^{3}$ Laboratoire COMETE, Caen, Normandie, France

${ }^{4}$ Research Institute for Sport and Exercise, University of Canberra, Canberra, Australian Capital Territory, Australia

\section{Correspondence to} Prof Sebastien Racinais, Research and Scientific Support Department, Aspetar Orthopaedic and Sports Medicine Hospital, 29222 Doha, Qatar;

sebastien.racinais@aspetar.com

Accepted 10 January 2020 Published Online First 28 January 2020

\section{ABSTRACT \\ Purpose Assess the health status and heat preparation strategies of athletes competing in a World Cycling Championships held in hot ambient conditions $\left(37^{\circ} \mathrm{C}\right.$, $25 \%$ relative humidity, wet-bulb-globe-temperature $27^{\circ} \mathrm{C}$ ) and monitor the medical events arising during competition.}

Methods 69 cyclists ( $9 \%$ of the world championships participants) completed a pre-competition questionnaire. IIInesses and injuries encountered by the Athlete Medical Centre (AMC) were extracted from the race reports.

Results $22 \%$ of respondents reported illness symptoms in the 10 days preceding the Championships. $57 \%$ of respondents had previously experienced heat-related symptoms (cramping most commonly) while 17\% had previously been diagnosed with exertional heat illness. $61 \%$ of the respondents had undergone some form of heat exposure prior to the Championships, with $38 \%$ acclimating for 5 to 30 days. In addition, several respondents declared to live in warm countries and all arrived in Qatar 5 days prior to their event. 96\% of the respondents used a pre-cooling strategy for the time trials and $74 \%$ did so before the road race $(p<0.001)$, with ice vests being the most common. The AMC assessed 46 injuries and 26 illnesses in total, with three cyclists diagnosed with heat exhaustion.

Conclusions The prevalence of previous heat illness in elite cyclists calls for team and event organisation doctors to be trained on heat illness management, including early diagnosis and rapid on-site cooling. Some cyclists had been exposed to the heat prior to the Championships, but few had a dedicated plan, calling for additional education on the importance of heat acclimation. Pre-cooling was widely adopted.

\section{INTRODUCTION}

The Union Cycliste Internationale (UCI), cycling's governing body, has recently established an Extreme Weather Protocol that incorporates a discussion between race officials, cyclists, team management and event organisers regarding any modifications to the race route. However, in the absence of epidemiological data on illness and injury in relation to environmental conditions, decisions concerning changes in racing remain difficult. The rare epidemiological studies linking environmental conditions to illness in athletes in competition indicate that the incidence of heat-related illness is very low in beach volleyball, ${ }^{1}$ and slightly higher in athletics. ${ }^{2}$ Such epidemiological data are missing in elite cyclists despite the fact that they can reach core temperatures well above $40^{\circ} \mathrm{C}$ during a world championship in the heat. ${ }^{3}$

Heat acclimation is widely considered the primary strategy to attenuate the decrement in performance and the risk of exertional heat illness when competing in warm environments. ${ }^{4} \mathrm{~A}$ recent study, however, revealed that only $15 \%$ of athletes competing in the International Association of Athletics Federations (IAAF) World Athletics Championships in Beijing 2015 reported heat acclimating for 5 to 30 days prior to an expected event in hot and humid conditions. ${ }^{2}$ Similarly, little is known regarding how elite cyclists prepare for these competitions, either in the weeks leading to the event (eg, heat acclimation), or the acute strategies used in the hours prior to, during or after the event.

Therefore, we aimed to assess the (i) health status and (ii) use of heat preparation strategies, as well as (iii) monitor the injury and illness burden at the Athlete Medical Centre (AMC) at the 2016 UCI Road World Championships in Qatar, in hot ambient conditions. Knowing the current status and practices of the elite athletes is the first step in identifying whether they are at risk of a health problem.

\section{METHODS}

This project uses two descriptive research designs: a survey and an observational method.

\section{Participants}

Sixty-nine cyclists competing in the team time trial (TTT), individual time trial (ITT) and/or road race (RR) at the 2016 UCI Road World Championship completed and returned the questionnaire (43 men and 26 women; 15 juniors, 15 under-23 (U23) and 39 elites; $\sim 9 \%$ of total participants). In addition, the medical cases of the 72 cyclists treated in the AMC were reviewed following the Championships. All procedures complied with the Declaration of Helsinki regarding human experimentation and written informed consent was obtained.

\section{Environmental conditions}

The environmental conditions during the Championships were hot (average temperature under direct sunlight $36.9^{\circ} \mathrm{C} \pm 2.8^{\circ} \mathrm{C}$ ), but dry (average relative humidity $24.6 \% \pm 15.6 \%$ ), corresponding to an 
outdoor wet-bulb-globe-temperature (WBGT) of $27.1^{\circ} \mathrm{C} \pm 2.4^{\circ} \mathrm{C}$. All courses were flat. The time trials lasted for $\sim 45 \mathrm{~min}$ whereas the RR lasted for $\sim 3$ hours $11 \mathrm{~min}$ and 5 hours $43 \mathrm{~min}$ for women and men, respectively.

\section{Questionnaire}

Participants completed a pre-competition heat strategy questionnaire in the days prior to competing in their event after arriving in Doha. The questionnaire was available in both UCI official languages (English and French). The questionnaire included the following sections: (i) demographic data, (ii) history of symptoms in the heat, (iii) history of exertional heat illness (EHI), (iv) heat acclimation (HA) strategy, (v) pre-existing condition(s) during the 10 days preceding the event, (vi) recovery strategy, (vii) pre-cooling strategy and (viii) hydration strategy. Sections vii and viii were repeated three times (for TTT, ITT and RR, respectively).

\section{Medical cases}

The number and type of illnesses and injuries encountered at the AMC were obtained from the race reports.

\section{Statistical analyses}

The data were coded and analysed using the SPSS software V.21.0 (SPSS Inc, Chicago, Illinois). A $\mathrm{X}^{2}$ test was used to determine the association of history of heat-related illnesses, symptoms, pre-travel acclimation strategy, pre-cooling strategies, hydration strategy and post-race recovery strategies with gender, race category (Elite, U23, Junior). A non-parametric Kruskal-Wallis test was used to compare the number of days that cyclists arrived before the race with type of first race. A p value $<0.05$ was used for statistical significance.

\section{RESULTS AND DISCUSSION}

Medical history and pre-competition health status History of heat-related symptoms and heat illness

The history of a previous diagnosis of EHI was not dependent on gender $(p=0.509)$ or race category $(p=0.885)$. The cyclists' history of heat-related symptoms is presented in table 1 . Most notably, the prevalence of symptoms was higher in men $(75 \%)$ than women $(28 \%)(\mathrm{p}<0.001)$. This was mainly associated with a higher rate of cramping $(58 \%$ vs $12 \%)(\mathrm{p}<0.001)$. Junior athletes reported fewer heat-related symptoms $(p=0.018)$. Seventeen per cent of the cyclists reported having been previously diagnosed with EHI (table 1). While self-reported data regarding clinical diagnoses may be inaccurate, cyclists were encouraged to discuss the questions with their medical staff if necessary. Overall, these data suggest that heat is a common issue for elite cyclists.

\section{Pre-existing illness in the 10 days preceding the race}

Twenty-two per cent of the respondents reported (any) illness symptoms in the 10 days preceding the World Championship (figure 1). This prevalence is higher than the IAAF World Championship where $13 \%$ of the athletes reported illness symptoms, especially considering that the IAAF questionnaire included the 4 weeks prior to the championship. ${ }^{5}$ It is important to note that the IAAF championships included a mixed profile of athletes (eg, track and field disciplines), whereas cycling can be considered as an endurance sport. In this context, the prevalence of illnesses before the UCI World Championships is similar to a large scale study on 7031 amateur long-distance runners where $19 \%$ reported pre-race illness symptoms. ${ }^{6}$ Thus, almost a quarter of respondents were symptomatic with the most common being headaches (56\%) and minor gastrointestinal symptoms (32\%).

\section{Pre-travel preparation and travel arrangements}

Training in the heat

Sixty-one per cent of the respondents had some form of heat exposure prior to the World Championships (table 1), following the recommendation that training in the heat is an important countermeasure to adopt before competing in the heat. ${ }^{4}$ Some riders adopted a strategy of between 1 and 4 days only; that is, a procedure likely too short for optimal adaptations. ${ }^{4}$ Thirty-eight per cent of the riders performed a dedicated HA period of at least 5 days. Of note, this proportion was more prevalent than in athletes participating in the IAAF Athletics World Championship (15\%). ${ }^{2}$ This might be partly explained by the fact that the IAAF championships were held in August, with most athletes already experiencing summer conditions whereas the UCI championships were held in October; and the IAAF athletes included jumpers, throwers and sprinters who may not need to acclimate from a performance perspective.

The 2015 IAAF athletes with a previous diagnosis of EHI were seven times more likely to train in the heat. ${ }^{2}$ However, the present study found no association between the HA strategy and a previous diagnosis of EHI (table 1). However, the pre-travel acclimation strategy in the current study depended on race category $(\mathrm{p}<0.001)$ with most elite cyclists spending at least 7 days training in the heat before travelling to Qatar (31\%) or living in a hot environment (23\%), while most juniors performed fewer than 7 days (80\%), and U23 had either none (57\%) or fewer than 7 days (21\%) only. This reinforces the need to inform nonelite (non-professional) categories about the importance of HA. Elite categories should also be aware of the benefits and limits of living in a hot environment as heat acclimation is not solely achieved by living in hot ambient conditions, but by training in the heat to induce an increase in core and skin temperature, as well as profuse sweating. ${ }^{4}$

Of note, the cyclists in the current study arrived in Qatar on average 5.4 \pm 2.5 days before competing in their event, affording most of them a heat acclimatisation stimulus, whether intended or not. Indeed, it has been shown that cyclists experience significant adaptation to the heat after training for only 1 week in Qatar, even while living in air-conditioned environments during the rest of the day. ${ }^{7}$

\section{Travel arrangements}

Only $6 \%$ of the respondents arrived in the 48 hours preceding their first race, while $58 \%$ arrived 3 to 6 days before and $23 \%$ arrived 7 to 10 days before their first race. The number of days between travel and the first race did not depend on the country travelled from, as most cyclists travelled from a European country at the end of their race season and crossed only two time zones (table 1) without considering jet lag in their travel plans. The combined management of jet lag, heat acclimation and tapering may however be more challenging during other events, such as the upcoming 2020 Tokyo Olympics.

\section{Race responses}

\section{Environmental conditions}

A total of 247 measures of ambient temperatures, 228 of relative humidity and 248 of WBGT were recorded from different locations along the course during the 8 days of the World Championship. The American College of Sports Medicine (ACSM) guidelines recommend to cancel all training and 
Table 1 History and strategy related to competing in the heat

\begin{tabular}{|c|c|c|c|c|c|c|}
\hline & Overall & Women & Men & Elite & U23 & Junior \\
\hline Number & 69 & 26 & 43 & 39 & 15 & 15 \\
\hline \multicolumn{7}{|c|}{ History of heat-related symptoms* } \\
\hline Cramping & $26(37.7)$ & $3(12.0) \dagger$ & $23(57.5)$ & $18(48.6)$ & $7(50.0)$ & $1(7.1) \ddagger \S$ \\
\hline Vomiting & $4(5.8)$ & $0(0.0)$ & $4(10.0)$ & $3(8.1)$ & $0(0.0)$ & $1(7.1)$ \\
\hline Nausea & $13(18.8)$ & $4(16.0)$ & $9(22.5)$ & $7(18.9)$ & $2(14.3)$ & $4(28.6)$ \\
\hline Severe headache & $10(14.5)$ & $2(8.0)$ & $8(20.0)$ & $6(16.2)$ & $3(21.4)$ & $1(7.1)$ \\
\hline Collapsing/fainting & $4(5.8)$ & $1(4.0)$ & $3(7.5)$ & $2(5.4)$ & $1(7.1)$ & $1(7.1)$ \\
\hline \multicolumn{7}{|c|}{ History of clinical exertional heat illness* } \\
\hline Exertional heat illness & $11(15.9)$ & $3(12.0)$ & $8(20.0)$ & $7(18.9)$ & $2(14.3)$ & $2(14.3)$ \\
\hline Dehydration & $5(7.2)$ & $2(8.0)$ & $3(7.5)$ & $4(10.8)$ & $0(0.0)$ & $1(7.1)$ \\
\hline Hyponatraemia & $1(1.4)$ & $1(4.0)$ & $0(0.0)$ & $0(0.0)$ & $0(0.0)$ & $1(7.1)$ \\
\hline Heat exhaustion & $2(2.9)$ & $0(0.0)$ & $2(5.0)$ & $1(2.7)$ & $1(7.1)$ & $0(0.0)$ \\
\hline Heat stroke & $8(11.6)$ & $2(8.0)$ & $6(15.0)$ & $5(13.5)$ & $2(14.3)$ & $1(7.1)$ \\
\hline \multicolumn{7}{|l|}{ Time zone crossed } \\
\hline 1 & $3(4.3)$ & $3(14.3) \dagger$ & $0(0.0)$ & $3(8.8)$ & $0(0.0)$ & $0(0.0)$ \\
\hline 2 & $46(66.7)$ & $17(81.0)$ & $29(85.3)$ & $28(82.4)$ & $7(70.0)$ & $11(100.0)$ \\
\hline 3 & $5(7.2)$ & $0(0.0)$ & $5(14.7)$ & $3(8.8)$ & $2(20.0)$ & $0(0.0)$ \\
\hline 10 & $1(1.4)$ & $1(4.8)$ & $0(0.0)$ & $0(0.0)$ & $1(10.0)$ & $0(0.0)$ \\
\hline \multicolumn{7}{|l|}{ No. of days arrived before race } \\
\hline$\leq 3$ & $18(26.1)$ & $10(43.5) \dagger$ & $8(19.5)$ & $7(19.4)$ & $6(40.0)$ & $5(38.5)$ \\
\hline $4-6$ & $23(33.3)$ & $4(17.4) \dagger$ & $19(46.3)$ & $15(41.7)$ & $4(26.7)$ & $4(30.8)$ \\
\hline $7-9$ & $16(23.2)$ & $4(17.4)$ & $12(29.3)$ & $7(19.4)$ & $5(33.3)$ & $4(30.8)$ \\
\hline 10 & $7(10.1)$ & $5(21.7) \dagger$ & $2(4.9)$ & $7(19.4)$ & $0(0.0)$ & $0(0.0)$ \\
\hline \multicolumn{7}{|l|}{ Heat training* } \\
\hline None & $15(21.7)$ & $2(7.7) \dagger$ & $13(30.2)$ & $6(15.4) \S$ & $8(57.1)$ & $1(6.7) \S$ \\
\hline $1-7$ days & $20(29.0)$ & $10(38.5)$ & $10(23.3)$ & $5(12.8)$ & $3(21.4)$ & $12(80)$ \\
\hline 8-14 days & $14(20.3)$ & $6(23.1)$ & $8(18.6)$ & $12(30.8)$ & $1(7.1)$ & $1(6.7) \neq$ \\
\hline Living in hot environment & $12(17.4)$ & $5(19.2)$ & $7(16.3)$ & $9(23.1)$ & $2(14.3)$ & $1(6.7)$ \\
\hline \multicolumn{7}{|l|}{ Post-race recovery strategy* } \\
\hline None & $5(7.2)$ & $2(7.7)$ & $3(7.0)$ & $0(0.0)$ & $1(6.7)$ & $4(26.7) \ddagger$ \\
\hline Active & $33(47.8)$ & $8(30.8) \dagger$ & $25(58.1)$ & $18(46.2)$ & $11(73.3)$ & $4(26.7) \S$ \\
\hline Stretching & $38(55.1)$ & $13(50.0)$ & $25(58.1)$ & $23(59.0)$ & $9(60.0)$ & $6(40.0)$ \\
\hline Massage & $53(76.8)$ & $20(76.9)$ & $33(76.7)$ & $33(84.6)$ & $11(73.3)$ & $9(60.0)$ \\
\hline Cold water immersion & $19(27.5)$ & $8(30.8)$ & $11(25.6)$ & $14(35.9)$ & $2(13.3)$ & $3(20.0)$ \\
\hline Hot water immersion & $3(4.3)$ & $2(7.7)$ & $1(2.3)$ & $1(2.6)$ & $1(6.7)$ & $1(6.7)$ \\
\hline Contrast immersion & $4(5.8)$ & $3(11.5)$ & $1(2.3)$ & $2(5.1)$ & $1(6.7)$ & $1(6.7)$ \\
\hline Compression clothing & 27 (39.1) & $15(57.7) \dagger$ & $12(27.9)$ & $15(38.5)$ & $7(46.7)$ & $5(33.3)$ \\
\hline Electro-stim & $7(10.1)$ & $4(15.4)$ & $3(7.0)$ & $1(2.6)$ & $2(13.3)$ & $4(26.7) \ddagger$ \\
\hline Other & $1(1.4)$ & $1(3.8)$ & $0(0.0)$ & $1(2.6)$ & $0(0.0)$ & $0(0.0)$ \\
\hline
\end{tabular}

Data as number (percentage of total).

* More than one possible choice, (proportions may not add to $100 \%$ ).

tSignificantly different than men.

‡Significantly different than Elite.

$\S$ Significantly different than U23, $p<0.05$.

U23, under-23.

competition when the WBGT reaches $32.3^{\circ} \mathrm{C}{ }^{8}$ This threshold (commonly characterised by a black flag) was reached in $1 \%$ of the measurements during the UCI World Championships. However, those measurements were from the car park and this threshold was never reached on the course or from the car following the race. As such, the UCI expert panel did not advise cancelling or shortening any of the races. Of note, the ACSM also suggests to cancel competition for continuous activity from a WBGT of $27.9^{\circ} \mathrm{C}$ (commonly characterised by orange and red flags), a threshold that was reached in 35\% of the measurements. However, the expert panel considered the cooling effect of the relative wind velocity while cycling at high speeds, which helps evaporative heat loss by removing the layer of saturated water vapour from the skin. ${ }^{9}{ }^{10}$

Although the UCI expert panel did not recommend any race cancellations, it did recommend increases in feeding and hydration opportunities. This recommendation was implemented by the local organisers and UCI commissaries, for example, by allowing car feedings during the ITT. Unfortunately, it was logistically not possible to increase the number of feeding stations on the race course lap circuit, a situation which was criticised by some cyclists. Such countermeasures should therefore be planned ahead of time as they may require additional resources and preparation. 




Figure 1 Prevalence of illness symptoms in the 10 days preceding the World Championships.

\section{Heat exhaustion}

The AMC assessed 46 injuries and 26 illness with six cyclists being transferred to the hospital for surgery $(n=3)$ or further examination $(n=3)$. In addition, three other cyclists were directly transferred from the road to the hospital following a crash (one head trauma, one knee trauma and one elbow trauma) without being assessed at the AMC. Only three cyclists were assessed for heat exhaustion, a mild form of EHI. They were treated with normal saline while receiving oral rehydration $(n=2)$ and oxygen $(\mathrm{n}=1)$. Another cyclist was assessed for exhaustion and received oral rehydration only. Although several finishers and medallists had a core temperature $>40^{\circ} \mathrm{C},{ }^{3}$ none of the athletes assessed for heat exhaustion had a rectal temperature $>40^{\circ} \mathrm{C}$, showing that no case of exertional heat stroke (the most severe form of EHI) was diagnosed by the AMC, although the time frame for assessing temperature is unknown. One rider was assessed for dehydration and potential arrhythmia, and was referred to the hospital for further examinations. The three recorded heat exhaustion cases over the 982 race starters at the UCI Cycling World Championships might appear lower than the five cases reported during the IAAF Athletic World Championships representing $1.6 \%$ of the athletes participating in the study. ${ }^{2}$ These numbers should however be treated with caution as the AMC was not located at the finish line and a larger proportion of injury and illness complaints might have been managed by the national team doctors without reporting to the AMC. As such, the current figure represents the number of encounters by the AMC facility to help future organisers plan for the necessary resources, rather than a true reflection of the illness burden associated with a World Championship event. Organiser should also aim to locate the AMC immediately after the finish line.

\section{Pre-race and post-race strategy \\ Pre-cooling strategy}

In line with recent meta-analyses concluding that pre-cooling can improve subsequent performance in the heat, ${ }^{11} 12$ most cyclists utilised a pre-cooling strategy. However, this was dependent on the event, with $96 \%$ of the respondents planning some form of pre-cooling before the TTT and ITT, versus $74 \%$ before the RR $(\mathrm{p}<0.001)$. There were also differences in cooling strategies (eg, fanning, ice vest, ice neck collar, whole body cold
Table 2 Pre-cooling and hydration strategy for each event

\begin{tabular}{|c|c|c|c|}
\hline & TTT & ITT & RR \\
\hline Number & 69 & 28 & 58 \\
\hline \multicolumn{4}{|l|}{ Pre-cooling strategy* } \\
\hline None & $1(1.4)$ & 1 (3.6) & $16(27.6) \dagger \ddagger$ \\
\hline Fanning & $12(17.4)$ & $12(42.9)$ & $4(6.9)$ \\
\hline Cold towel & $13(18.8)$ & $17(60.7)$ & $22(37.9)$ \\
\hline Neck collar & $10(14.5)$ & $9(32.1)$ & $9(15.5)$ \\
\hline Whole body cold water immersion & $5(7.2)$ & $0(0.0)$ & $4(6.9)$ \\
\hline Leg CWI & $2(2.9)$ & $2(7.1)$ & $6(10.3)$ \\
\hline Ice vest & $19(27.5)$ & $26(92.9)$ & $39(67.2)$ \\
\hline Ice slurry & $9(13.0)$ & $4(14.3)$ & $5(8.6)$ \\
\hline Other & - & - & $1(1.7)$ \\
\hline \multicolumn{4}{|l|}{ Hydration strategy } \\
\hline Less than $0.5 \mathrm{~L}$ & 16 (69.6) & $16(66.7)$ & $1(1.6)$ \\
\hline 0.5 to $1 \mathrm{~L}$ & $4(17.4)$ & $6(25.0)$ & - \\
\hline 1 to $1.5 \mathrm{~L}$ & $1(4.3)$ & $2(8.3)$ & $2(3.3)$ \\
\hline 1.5 to $2 \mathrm{~L}$ & - & - & $3(4.9)$ \\
\hline More than $2 \mathrm{~L}$ & $2(8.7)$ & - & $55(90.2)$ \\
\hline \multicolumn{4}{|l|}{ Preferred fluid } \\
\hline Water & $9(13.0)$ & $15(53.6)$ & 56 (96.6) \\
\hline Electrolyte & 19 (27.5) & $15(53.6)$ & $42(72.4)$ \\
\hline Carb & $10(14.5)$ & $9(32.1)$ & $40(69)$ \\
\hline Ice slurry & $2(2.9)$ & $1(3.6)$ & $6(10.3)$ \\
\hline Other & - & - & - \\
\hline
\end{tabular}

Data as number (percentage of total).

${ }^{*}$ More than one possible choice, (proportions may not add to $100 \%$ ).

†Significantly different than TTT.

¥Significantly different than ITT, $p<0.05$.

CWI, cold water immersion; ITT, individual time trial; RR, road race; $T T T$, team time trial.

water immersion (CWI) and ice slurry) (all $\mathrm{p}<0.05$, table 2). This wide-spread use of pre-cooling call for event organisers to provide the necessary facilities such as fan, fridge to accommodate cold vest or power supply for ice slurry machine. This may appear as beyond the duties of the organiser but this would help ensuring equities between countries with and without a strong logistical support.

Eight per cent of the respondents planned on having ice slurry during the races itself, irrespective of the event $(p=0.597)$. The prevalence of leg CWI was 9\%, irrespective of the event $(\mathrm{p}=0.913)$. Leg CWI requires some logistical planning and it is likely not suitable before a time trial as decreasing muscle temperature limits sprint performance and likely power output during the first minutes of a time trial. ${ }^{412}$ An easily applicable pre-cooling strategy is the use of cold towels, ${ }^{4}$ which were used before all race types. The differences in strategies between the TT and RR are likely due to the event duration and warm-up type. Cyclists typically perform an intense stationary warm-up before starting a TT during which cooling strategies such as wearing an ice vest and ingesting ice slurry might attenuate the rise in core and skin temperature. ${ }^{11}$ More cyclists planned on using ice vests and cold towels (ie, two portable solutions) before the ITT than TTT, whereas more planned CWI and ice slurry (ie, two solutions requiring logistical supports) before the TTT than ITT. The differences between the TTT and ITT may relate to the TTT being a professional trade team event where the athletes were benefiting from their usual sports science support team.

The different prevalence of pre-cooling strategy used between the RR and TT was specific to men. In women, $96 \%$ of 
respondents planned on using pre-cooling before the RR, versus $57 \%$ of men $(p=0.001)$. This result showed a different trend in cyclists than observed during the IAAF Athletics World Championships where more men (52\%) than women (42\%) planned to use pre-cooling. ${ }^{2}$ This gender difference may be due to the limited sample size and the main results remain that most cyclists pre-cool before a cycling race in the heat.

\section{Hydration strategy}

The elevated thermal strain associated with exercising in hot and humid environments increases fluid and electrolyte losses. It has been reported that athletes may tolerate up to $4 \%$ of body mass loss during real-world time trials without experiencing a performance impairment, ${ }^{13}$ but dehydration is well known to exacerbate heat strain. ${ }^{14} \mathrm{~A}$ recent meta-analyses demonstrated that fluid ingestion is the most efficient countermeasure to attenuate the rise in core temperature when exercising in the heat and is the third most effective countermeasure, after aerobic fitness and heat acclimation, to improve endurance performance in the heat. ${ }^{15}$ Different hydration strategies were observed between events; partly explained by the fact that cyclists used only one bottle during the time trials (electrolytes for most riders), whereas they used several bottles during the RR (electrolytes and water) (table 2). Furthermore, $66 \%$ of the respondents planned to include carbohydrate in their drinks during the RR, but only $42 \%$ and $32 \%$ during the TTT and ITT $(p=0.007)$. It is important to note that this figure does not include additional consumption of energy bars, gels or other sources of food or fluids.

\section{Post-race recovery strategy}

Ninety-two per cent of respondents planned on using one or several recovery strategies (table 1 ). This high prevalence is in line with the $89 \%$ reported during the IAAF Athletics World Championships. ${ }^{2}$ Despite limited research, massage was the most frequently utilised recovery strategy (77\%), likely as it is anecdotally believed to have numerous benefits. ${ }^{16}$ Stretching $(55 \%)$ and active cool down (48\%) were also prevalent. Thirtynine per cent of respondents planned on utilising compression clothing, with indications that it may improve recovery after a cycling time trial. ${ }^{17}$ One-third of the respondents (34\%) planned on using water immersion, mainly cold (CWI, 28\%), but also hot $(4 \%)$ or contrasted $(6 \%)$. While being superior to passive recovery or rest, there are no clear differences between the benefits of these different immersion techniques. ${ }^{18}$

The proportion of cyclists planning on using a recovery strategy was equivalent in men and women $(p=1.000)$, but the nature of recovery differed between genders. Only $31 \%$ of women planned to actively recover compared with $60 \%$ of men $(\mathrm{p}=0.026)$. Conversely, $60 \%$ of women cyclists planned to use compression clothing while this percentage decreased to $29 \%$ in men ( $\mathrm{p}=0.023)$. The prevalence for the other recovery techniques was equivalent between genders. This observation partly contrasts with a previous report showing that women athletes during the IAAF World Championship were planning recovery strategies more frequently than men, with a greater use of massage. ${ }^{2}$ This difference is likely due to the fact that massage is the primary recovery technique in cycling, independent of gender.

\section{Limitations}

The environmental conditions were hot but not extreme due to a low relative humidity. Medical encounters due to heat illness may be higher in more extreme/humid conditions or in a different sport (eg, with limited airflow). Although the response rate in the current study is low (9\%), it does cover the different categories (ie, men and women; juniors, U23 and elites).

\section{CONCLUSION}

The current results show a history of heat-related symptoms (57\%) and some incidents of EHI (17\%) in elite cyclists. This highlights the need for team doctors to be aware of the four 'golden rules' of heat stroke management (ie, early recognition, early diagnosis, rapid cooling, on-site cooling). ${ }^{19}$ Our study showed that most elite cyclists use a pre-cooling strategy before competing in the heat, suggesting a need for race organisers to provide supporting facilities such as ice, cold-water bath, ice slurry machine, freezer for ice vest storage, etc. Lastly, $61 \%$ of the respondents had some form of heat exposure prior to the event, but only $38 \%$ had a dedicated heat acclimation period of 5 to 30 days, suggesting a need for additional information dissemination on the importance of heat acclimation.

\section{What are the findings?}

- A quarter of the cyclists who completed a pre-competition questionnaire reported illness symptoms in the 10 days preceding the Union Cycliste Internationale 2016 Road World Championships.

- Sixty-one per cent of the respondents had some form of heat exposure prior to the event; $38 \%$ had a dedicated heat acclimation period of 5 to 30 days.

- Almost all time trial participants and $74 \%$ of road race participants planned on using a pre-cooling strategy before competing in the heat.

\section{How might it impact on clinical practice in the future?}

- There is still a need to educate athletes on the importance of heat acclimation enough in advance of international events as numerous participants did not follow the recommendation of 2 weeks of heat acclimation.

- Logistical requirements need to be identified in advance to properly support the needs of cyclists (eg, pre-cooling facilities).

Twitter Sebastien Racinais@ephysiol, David Nichols @dave_vo2project, Abdulaziz Farooq @azizfar and Julien D Périard @DrJPeriard

Acknowledgements The authors thank the UCl and the medical commission for their endorsement and operational support. The authors thank the local organising committee for their operational and financial support.

Contributors SR, TB, YOS and JP designed the study. SR, DN, GT and SM collected the data. SR, DN and AF analysed of the data. All authors made substantial contributions to data interpretation. SR, JP and DN drafted the article. All authors revised the manuscript and approved the final version.

Funding This study was supported by the 2016 UCI Road World Championships organising committee.

Competing interests None declared.

Patient consent for publication Not required.

Ethics approval The study was approved by the Anti-Doping Lab Qatar Institutional Review Board (E2016000162) and conformed to the current Declaration of Helsinki guidelines.

Provenance and peer review Not commissioned; externally peer reviewed.

Data availability statement Data are available upon reasonable request.

\section{ORCID iDs}

Sebastien Racinais http://orcid.org/0000-0003-0348-4744

Abdulaziz Faroog http://orcid.org/0000-0002-9162-4948 


\section{REFERENCES}

1 Bahr R, Reeser JC. New guidelines are needed to manage heat stress in elite sports-The Fédération Internationale de Volleyball (FIVB) Heat Stress Monitoring Programme. Br J Sports Med 2012;46:805-9.

2 Périard JD, Racinais S, Timpka T, et al. Strategies and factors associated with preparing for competing in the heat: a cohort study at the 2015 IAAF world athletics Championships. Br J Sports Med 2017; 51:264-70.

3 Racinais S, Moussay S, Nichols D, et al. Core temperature up to $41.5^{\circ} \mathrm{C}$ during the $\mathrm{UCl}$ road cycling world Championships in the heat. Br J Sports Med 2019;53:426-9.

4 Racinais S, Alonso JM, Coutts AJ, et al. Consensus recommendations on training and competing in the heat. Br J Sports Med 2015;49:1164-73.

5 Timpka T, Jacobsson J, Bargoria V, et al. Preparticipation predictors for Championship injury and illness: cohort study at the Beijing 2015 international association of athletics Federations world Championships. Br J Sports Med 2017;51:271-6.

6 Van Tonder A, Schwellnus M, Swanevelder S, et al. A prospective cohort study of 7031 distance runners shows that 1 in 13 report systemic symptoms of an acute illness in the 8-12 day period before a race, increasing their risk of not finishing the race 1.9 times for those runners who started the race: SAFER study IV. Br J Sports Med 2016;50:939-45.

7 Racinais S, Périard JD, Karlsen A, et al. Effect of heat and heat acclimatization on cycling time trial performance and pacing. Med Sci Sports Exerc 2015;47:601-6.

8 Armstrong LE, Casa DJ, Millard-Stafford M, et al. American College of sports medicine position stand. exertional heat illness during training and competition. Med Sci Sports Exerc 2007;39:556-72.

9 Saunders AG, Dugas JP, Tucker R, et al. The effects of different air velocities on heat storage and body temperature in humans cycling in a hot, humid environment. Acta Physiol Scand 2005;183:241-55.
10 Nybo L. Cycling in the heat: performance perspectives and cerebral challenges. Scand J Med Sci Sports 2010;20:71-9.

11 Bongers CCWG, Thijssen DHJ, Veltmeijer MTW, et al. Precooling and percooling (cooling during exercise) both improve performance in the heat: a meta-analytical review. Br J Sports Med 2015;49:377-84.

12 Tyler CJ, Sunderland C, Cheung SS. The effect of cooling prior to and during exercise on exercise performance and capacity in the heat: a meta-analysis. Br J Sports Med 2015;49:7-13.

13 Goulet EDB. Effect of exercise-induced dehydration on endurance performance: evaluating the impact of exercise protocols on outcomes using a meta-analytic procedure. Br J Sports Med 2013;47:679-86.

14 Sawka MN, Cheuvront SN, Kenefick RW. Hypohydration and human performance: impact of environment and physiological mechanisms. Sports Med 2015:45:51-60.

15 Alhadad SB, Tan PMS, Lee JKW. Efficacy of heat mitigation strategies on core temperature and endurance exercise: a meta-analysis. Front Physiol 2019;10:71.

16 Weerapong P, Hume PA, Kolt GS. The mechanisms of massage and effects on performance, muscle recovery and injury prevention. Sports Med 2005;35:235-56.

17 de Glanville KM, Hamlin MJ. Positive effect of lower body compression garments on subsequent $40-\mathrm{kM}$ cycling time trial performance. J Strength Cond Res 2012;26:480-6.

18 Bieuzen F, Bleakley CM, Costello JT. Contrast water therapy and exercise induced muscle damage: a systematic review and meta-analysis. PLoS One 2013;8:e62356-15.

19 Racinais S, Casa D, Brocherie F, et al. Translating science into practice: the perspective of the Doha 2019 IAAF world Championships in the heat. Front. Sports Act. Living 2019;1:39. 